\title{
Adenosine activates thermogenic adipocytes
}

\author{
Cell Research (2015) 25:155-156. doi:10.1038/cr.2014.157; published online 2 December 2014
}

\begin{abstract}
Brown or beige fat activation can cause potent anti-obesity and antidiabetic effects. In a study recently published in Nature, Gnad et al. show that adenosine is a novel activator of brown and beige fat that acts through the $A_{2 A}$ receptor.
\end{abstract}

Brown fat is a thermogenic type of adipose tissue containing abundant mitochondria and multilocular lipid droplets, and is uniquely suited and required for cold tolerance and body weight control [1]. Upon brown fat activation, intracellular cyclic AMP (cAMP) levels rise rapidly, leading to lipolysis and upregulation of uncoupling protein 1 (UCP1) which initiates uncoupling of mitochondrial respiration and heat generation [2,3]. Additionally, beige or brite fat is a form of white fat that can be "browned" and induced to engage in respiratory uncoupling similarly as brown fat. Activated brown and beige fat consume substantial amounts of metabolic substrate, leading to powerful anti-obesity and anti-diabetic effects in mice. Adult humans possess brown and beige fat, making these tissues potentially important targets for treating obesity and metabolic diseases [4]. Activation of brown and beige fat occurs through several mechanisms including cold, adrenergic signaling, and hormone signaling [4]. However, a critical question remains of which pathway is altered therapeutically, as targeting these known activation pathways may cause discomfort, have undesirable side effects, or be insufficient alone to exert anti-obesity effects in humans. Thus, the need to discover novel pathways for brown and beige fat thermogenic activation persists.
Adenosine is a purine nucleoside that alters cAMP signaling in several tissues $[5,6]$. There is a ubiquitous presence of adenosine in the extracellular space, and adenosine levels can be increased extracellularly or intracellularly through conversion of adenine nucleotides. Adenosine binds to four P1 G-proteincoupled receptor subtypes, which include the inhibitory receptors $\mathrm{A}_{1}$ and $\mathrm{A}_{3}$ and the stimulatory receptors $\mathrm{A}_{2 \mathrm{~A}}$ and $A_{2 B}$ [7]. Inhibitory receptor activation leads to increased adenylate cyclase activity and decreased cAMP, while stimulatory receptor activation has the opposite effect. The distribution of the adenine receptor subtypes varies widely by tissue and species, and the response is dependent on the level of receptor expression, thus the actions of adenosine can be highly disparate depending on the tissue context [8].

In adipose tissue, adenosine was previously found to inhibit cAMP production in studies performed in hamster and rat, where oxygen consumption and lipolysis also decreased $[9,10]$. In a study recently published in Nature, Gnad et al. [11] show that adenosine activates lipolysis and the thermogenic program in brown and white human and murine adipocytes (Figure 1). This phenomenon occurs at significantly lower concentrations of adenosine in brown adipocytes than in white adipocytes. The difference is accounted for by differential expression of the $A_{2 A}$ receptor, which is highly expressed in human and murine brown fat relative to white fat. It is also due to the ratio of expression compared to the inhibitory $\mathrm{A}_{1}$ receptor, which is expressed at low levels in brown fat compared to white fat. Moreover, the authors show that hamster brown fat expresses almost equal amounts of $\mathrm{A}_{1}$ and $\mathrm{A}_{2 \mathrm{~A}}$ receptors, explaining the lack of activating effects of adenosine in those cells.

The determination of the source of the adenosine driving brown fat activation was also pursued. Adenosine could be derived from breakdown of ATP released from sympathetic neurons and brown adipocytes, or from brown adipocyte efflux of adenosine itself. The authors find that activated brown fat both releases adenosine through efflux and converts extracellular ATP to adenosine, as inhibition of the ecto5'-nucleotidase CD73, which converts ATP to adenosine, only partially blocks the adenosine increase. Noradrenaline also enhances adenosine release from brown fat without any change in extracellular ATP, suggesting an intracellular crosstalk between catecholamine and adenosine signaling.

The author further show that $\mathrm{A}_{2 \mathrm{~A}}$ receptor knockout mice exposed to cold exhibit defective thermogenesis, oxygen consumption, and lipolysis, demonstrating the importance of the $\mathrm{A}_{2 \mathrm{~A}}$ receptor in mediating the thermogenic response. Moreover, pharmacological activation with an $\mathrm{A}_{2 \mathrm{~A}}$ receptor agonist increases oxygen consumption in mice, while inhibition with a specific $\mathrm{A}_{2 \mathrm{~A}}$ antagonist decreases cold-induced oxygen consumption. $\mathrm{A}_{2 \mathrm{~A}}$ receptor agonism acts synergistically with noradrenaline in driving lipolysis, revealing an additive thermogenic effect of adenosine with catecholamine signaling. $\mathrm{An}_{2 \mathrm{~A}}$ agonist protects against diet-induced obesity in mice, leading to increases in lean mass, oxygen consumption, glucose 


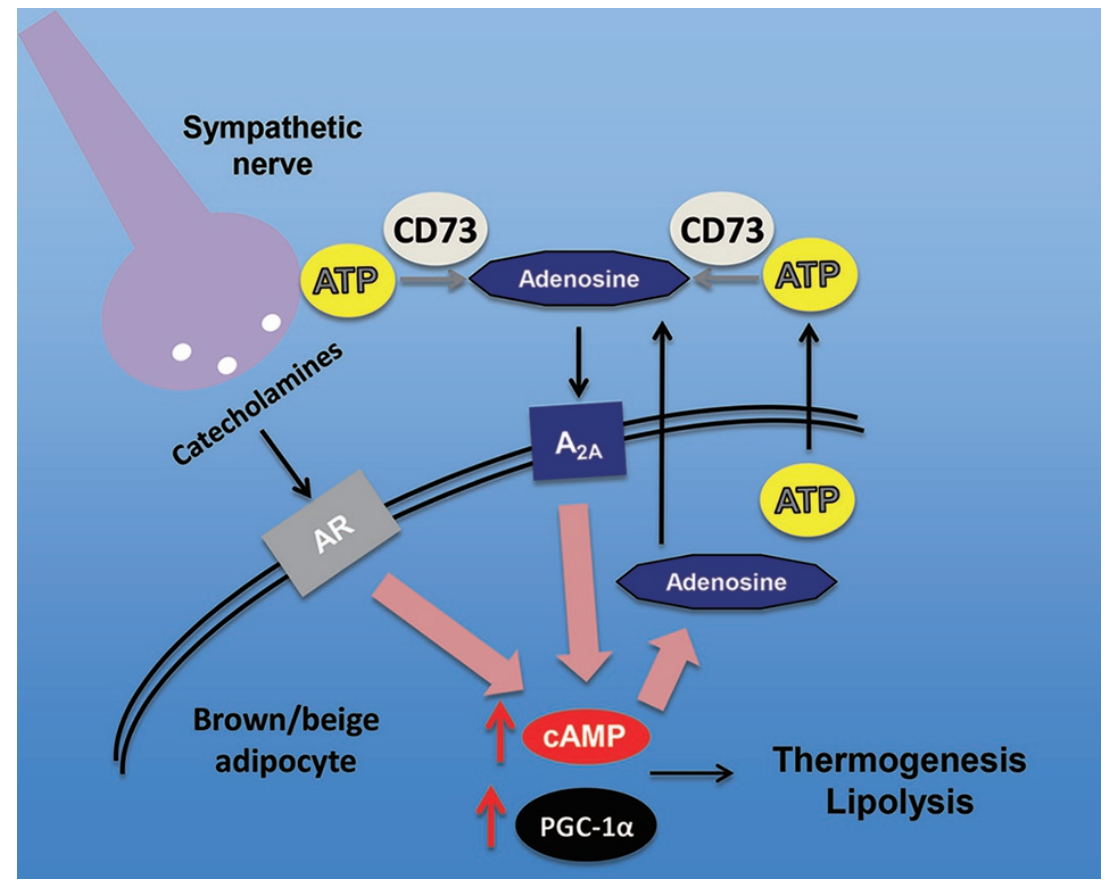

Figure 1 Adenosine activates brown or beige adipocytes through the $A_{2 A}$ receptor. Adenosine activates the $A_{2 A}$ receptor, leading to increased cAMP- and PGC- $1 \alpha-$ dependent signaling that enhances thermogenesis and lipolysis in brown and beige adipocytes. Crosstalk occurs with adrenergic signaling, which increases cAMP and adenosine levels. Adenosine is released both through efflux from brown adipocytes and through breakdown of extracellular ATP by the ecto-5'-nucleotidase CD73. AR, adrenergic receptor.

tolerance, and browning of white fat, which signifies the potential therapeutic importance of adenosine signaling. Importantly, overexpression of the $\mathrm{A}_{2 \mathrm{~A}}$ receptor in inguinal white fat increases its browning, indicating that bolstering of $\mathrm{A}_{2 \mathrm{~A}}$ adenosine receptor expression is sufficient to initiate a thermogenic response in the presence of physiological concentration of adenosine.

This study reveals an endogenous purinergic signaling pathway that activates brown and beige fat. The critical signaling effector involved is cAMP, therefore the mechanism of activation is similar to those of other agents that act through the sympathetic nervous system or adrenergic signaling. It is unclear whether adenosine would contribute any additional anti-obesity effects than what has already been achieved with these other agents.

While this study demonstrates substantial effects of adenosine on thermogenesis, further studies would better elucidate the specificity toward the $\mathrm{A}_{2 \mathrm{~A}}$ receptor in adipocytes. Treatment of $\mathrm{A}_{2 \mathrm{~A}}$ knockout mice with the receptor agonist would help demonstrate whether there are effects of the agonist independent of the $\mathrm{A}_{2 \mathrm{~A}}$ receptor in vivo. Additionally, adipose tissue-specific $A_{2 A}$ knockout mice would reveal whether there are any phenotypic contributions from knockout in other tissues.

Another question is whether $\mathrm{A}_{2 \mathrm{~A}}$ receptor agonism could be used chronically as an anti-obesity therapy in the clinic, since adenosine receptors are expressed in many tissues. This

study did not explore the potentially confounding effects of $A_{2 A}$ receptor agonism on tissues such as the brain or heart, including complications from vasodilation or cardiotoxicity. Indeed, $\mathrm{A}_{2 \mathrm{~A}}$ receptor antagonists have been used in clinical trials for Parkinson's disease, highlighting the possible adverse effects of $\mathrm{A}_{2 \mathrm{~A}}$ receptor agonism on the brain. The effect of adenosine on lipolysis also raises the question of whether this pathway is involved in the lipoatrophy caused by nucleoside analogues used as anti-retroviral drugs. Nevertheless, this work adds important information about a cellular signaling pathway that may be leveraged in finding new therapies for obesity and metabolic diseases.

\section{Amy K Rines ${ }^{1}$, Francisco Verdeguer ${ }^{1}$, Pere Puigserver ${ }^{1}$}

${ }^{1}$ Department of Cancer Biology, Dana-Farber Cancer Institute and Department of Cell Biology, Harvard Medical School, Boston, MA 02215, USA Correspondence: Pere Puigserver E-mail: pere_puigserver@dfci.harvard.edu

\section{References}

1 Lowell BB, Flier JS. Annu Rev Med 1997; 48:307-316.

2 Lowell BB, Spiegelman BM. Nature 2000; 404:652-660.

3 Puigserver P, Wu Z, Park CW, et al. Cell 1998; 92:829-839.

4 Kajimura S, Saito M. Аnпи Rev Physiol 2014; 76:225-249.

5 Fredholm BB, Jonzon B, Lindgren E, et al. J Neurochem 1982; 39:165-175.

6 Kleppisch T, Nelson MT. Proc Natl Acad Sci USA 1995; 92:12441-12445.

7 Fredholm BB, IJzerman AP, Jacobson KA, et al. Pharmacol Rev 2011; 63:1-34.

8 Johansson B, Halldner L, Dunwiddie TV, et al. Proc Natl Acad Sci USA 2001; 98:94079412.

9 Fain JN, Pointer RH, Ward WF. J Biol Chem 1972; 247:6866-6872.

10 Schimmel RJ, McCarthy L. Am J Physiol 1984; 246:C301-C307.

11 Gnad T, Scheibler S, von Kügelgen I, et al. Nature 2014; 516:395-399. 